\title{
Interpretation of angular distributions of Z-boson production
}

\section{Jen-Chieh Peng*}

Department of Physics, University of Illinois at Urbana-Champaign, Urbana, IL 61801, USA

E-mail: jcpengeillinois.edu

\section{Wen-Chen Chang}

Institute of Physics, Academia Sinica, Taipei 11529, Taiwan

E-mail: Changwc@phys.sinica.edu.tw

\section{Randall Evan McClellan}

Thomas Jefferson National Accelerator Facility, Newport News, VA 23606, USA

E-mail: randallm@jlab.org

\section{Oleg Teryaev}

Bogoliubov Laboratory of Theoretical Physics, JINR, 141980 Dubna, Russia

E-mail: teryaev@theor.jinr.ru

We present an intuitive approach for describing the angular distributions of leptons produced in the Drell-Yan process and Z-boson production in hadron-hadron collisions. We show that this approach can describe the pronounced transverse-momentum dependence of the $\lambda$ and $v$ parameters, observed at the $\gamma^{*} / Z$ production at Tevatron and LHC, very well. The violation of the Lam-Tung relation is attributed to the mis-alignment of the hadronic plane and the quark plane in this approach, and can be well described in this approach.

QCD Evolution 2016

May 30-June 03, 2016

National Institute for Subatomic Physics (Nikhef), Amsterdam

\footnotetext{
* Speaker.
} 


\section{Introduction}

The lepton angular distributions in the Drell-Yan process potentially carry important information on the dynamics of the reaction and on the partonic structures of the colliding hadrons. In the naive Drell-Yan model [1], it was predicted that quark and antiquark annihilat into a transversely polarized photon, leading to a $1+\cos ^{2} \theta$ lepton angular distribution. While this prediction was soon confirmed by the earliest Drell-Yan experiments [2], a more general angular distribution expression is expected when the intrinsic transverse momentum of the partons and/or the QCD effects are included, namely [3],

$$
\frac{d \sigma}{d \Omega} \propto 1+\lambda \cos ^{2} \theta+\mu \sin 2 \theta \cos \phi+\frac{v}{2} \sin ^{2} \theta \cos 2 \phi,
$$

where $\theta$ and $\phi$ refer to the polar and azimuthal angle, of the $l^{-}$in the dilepton rest frame. The azimuthal symmetry in the collinear naive Drell-Yan model is lost due to the finite transverse momentum $\left(q_{T}\right)$ of the dilepton. While $\lambda=1, \mu=v=0$ for the naive Drell-Yan model, the finite value of $q_{T}$ leads to $\lambda \neq 1$ and $\mu, v \neq 0$. Nevertheless, it was pointed out by Lam and Tung that the deviation of $\lambda$ from 1 is related to the deviation of $v$ from zero through the relation, $1-\lambda=2 v[3]$. This "Lam-Tung" relation was also predicted to be insenstive to QCD correction [ [

First measurements of the lepton polar and azimuthal angular distributions were carried out by the CERN NA10 [5] and the Fermilab E615 [6] Collaborations. Surprisingly large violations of the Lam-Tung relation were observed, prompting many novel interpretations. In particular, Boer showed that the presence of the Boer-Mulders function, can explain the violation of the Lam-Tung relation [7]. Results from a Drell-Yan experiment [8] using proton beam were also shown to be consistent with this interpretation. Interesting recent developments include the measurements of the lepton angular distribution of $Z$-boson production in $p-\bar{p}$ collision by the CDF Collaboration at the Tevatron [9] and in $p-p$ collisions by the CMS Collaboration at the Large Hadron Collider [10]. Both the CDF and CMS data show striking $q_{T}$ dependencies for $\lambda$ and $v$. Moreover, the highstatistics CMS measurement clearly shows that the Lam-Tung relation is violated even at the large transverse momentum region ( $p_{T}$ up to $\sim 300 \mathrm{GeV}$ ) where effect from the Boer-Mulders function should be negligible.

We present an interpretation for the CMS and CDF results on the $q_{T}$ dependencies of the angular distribution coefficients $\lambda$ and $v$, as well as the origin for the violation of the Lam-Tung relation. A more detailed discussion can be found in a recent publication [11].

\section{Drell-Yan angular distribution}

The angular distribution of the leptons is usually expressed in the rest frame of $\gamma^{*} / Z$. In the Collins-Soper (C-S) frame [12], the $\hat{x}$ and $\hat{z}$ axes lie in the hadron plane formed by the colliding hadrons and the $\hat{z}$ axis bisects the momentum vectors of the two hadrons (see Figure 11). Another plane, called the quark plane, is formed by the axis of the collinear $q$ and $\bar{q}$ which combines into the $\gamma^{*} / Z$ and the $\hat{z}$ axis. The momentum unit vector of $q$ is defined as $\hat{z}^{\prime}$, which has polar and azimuthal angles $\theta_{1}$ and $\phi_{1}$, as shown in Fig. 1. Finally, the back-to-back $l^{-}$and $l^{+}$, together with the $\hat{z}$ axis, form the lepton plane. The lepton $l^{-}\left(e^{-}\right.$or $\left.\mu^{-}\right)$from the $\gamma^{*} / Z$ decay have polar and azimuthal 
angle in the C-S frame $\theta$ and $\phi$ in the C-S frame, as shown in Fig. 1. For any given values of $\theta$ and $\phi, \theta_{1}$ and $\phi_{1}$ can vary over a range of values.

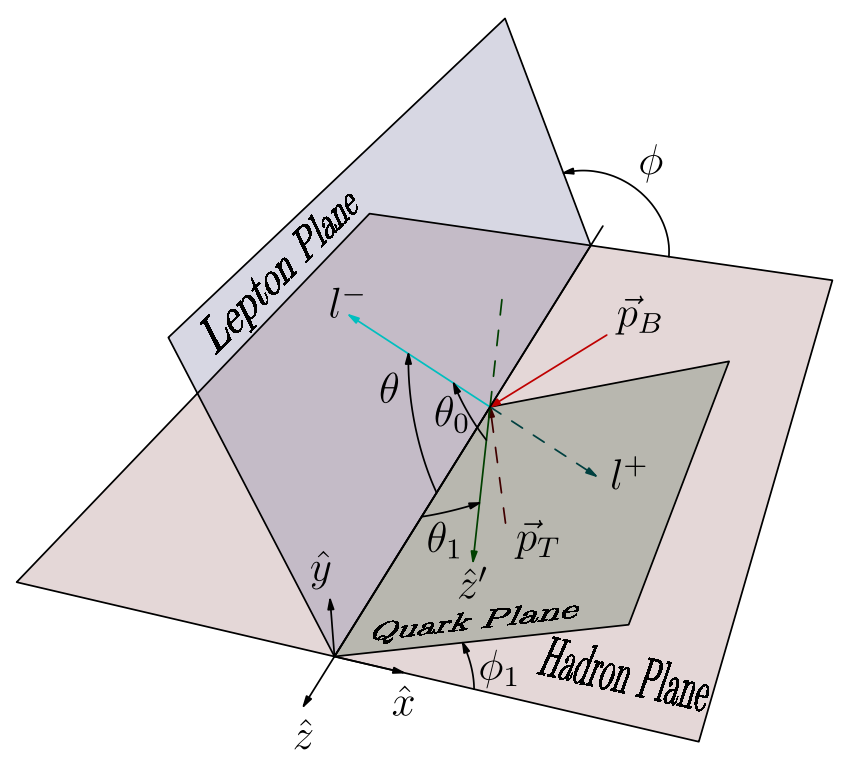

Figure 1: Definition of the Collins-Soper coordinates, the hadron plane, the lepton plane, and the quark plane.

Taking into account the contribution of parity-violating coupling involving the $Z$ boson, the general angular distribution for $\gamma^{*} / Z$ production is given as [10]

$$
\begin{aligned}
\frac{d \sigma}{d \Omega} & \propto\left(1+\cos ^{2} \theta\right)+\frac{A_{0}}{2}\left(1-3 \cos ^{2} \theta\right)+A_{1} \sin 2 \theta \cos \phi+\frac{A_{2}}{2} \sin ^{2} \theta \cos 2 \phi \\
& +A_{3} \sin \theta \cos \phi+A_{4} \cos \theta+A_{5} \sin ^{2} \theta \sin 2 \phi+A_{6} \sin 2 \theta \sin \phi+A_{7} \sin \theta \sin \phi .
\end{aligned}
$$

We show now how this expression can be derived. First, helicity conservation in the $q \bar{q} \rightarrow l^{-} l^{+}$ reaction implies that the angular distribution of $l^{-}$must be azimuthally symmetric with respect to the $\hat{z}^{\prime}$ axis with the following polar angular dependence:

$$
\frac{d \sigma}{d \Omega} \propto 1+a \cos \theta_{0}+\cos ^{2} \theta_{0}
$$

The forward-backward asymmetry coefficient $a$ comes from the parity-violating coupling to the $Z$ boson, and $\theta_{0}$ is the angle between the $l^{-}$momentum vector and $\hat{z}^{\prime}$, as shown in Fig. 1. To go from Eq. 2.2 to Eq. 2.1, we note that $\cos \theta_{0}$ satisfies the relation:

$$
\cos \theta_{0}=\cos \theta \cos \theta_{1}+\sin \theta \sin \theta_{1} \cos \left(\phi-\phi_{1}\right) .
$$

Substituting Eq. 2.3 into Eq.2.2, we obtain

$$
\begin{aligned}
\frac{d \sigma}{d \Omega} \propto & \left(1+\cos ^{2} \theta\right)+\frac{\sin ^{2} \theta_{1}}{2}\left(1-3 \cos ^{2} \theta\right)+\left(\frac{1}{2} \sin 2 \theta_{1} \cos \phi_{1}\right) \sin ^{2} \theta \cos 2 \phi \\
& +\left(a \sin \theta_{1} \cos \phi_{1}\right) \sin \theta \cos \phi+\left(a \cos \theta_{1}\right) \cos \theta+\left(\frac{1}{2} \sin ^{2} \theta_{1} \sin 2 \phi_{1}\right) \sin ^{2} \theta \sin 2 \phi \\
& +\left(\frac{1}{2} \sin 2 \theta_{1} \sin \phi_{1}\right) \sin 2 \theta \sin \phi+\left(a \sin \theta_{1} \sin \phi_{1}\right) \sin \theta \sin \phi .
\end{aligned}
$$


From Eq.2.1 and Eq. 2.4 one can express $A_{0}$ to $A_{7}$ in terms of $\theta_{1}, \phi_{1}$ and $a$ as follows:

$$
\begin{aligned}
A_{0} & =\left\langle\sin ^{2} \theta_{1}\right\rangle & A_{1} & =\frac{1}{2}\left\langle\sin 2 \theta_{1} \cos \phi_{1}\right\rangle \\
A_{2} & =\left\langle\sin ^{2} \theta_{1} \cos 2 \phi_{1}\right\rangle & A_{3} & =\left\langle a \sin \theta_{1} \cos \phi_{1}\right\rangle \\
A_{4} & =\left\langle a \cos \theta_{1}\right\rangle & A_{5} & =\frac{1}{2}\left\langle\sin ^{2} \theta_{1} \sin 2 \phi_{1}\right\rangle \\
A_{6} & =\frac{1}{2}\left\langle\sin 2 \theta_{1} \sin \phi_{1}\right\rangle & A_{7} & =\left\langle a \sin \theta_{1}\right\rangle\left\langle\sin \phi_{1}\right\rangle .
\end{aligned}
$$

Equation 2.5] is a generalization of an earlier work [13] which considered the special case of $\phi_{1}=0$ and $a=0$. The $\langle\cdot \cdot\rangle$ in Eq. 2.5 is a reminder that the measured values of $A_{n}$ are averaged over the event sample. A comparison of Eq. 1.1 and Eq. 2.1 gives

$$
\lambda=\frac{2-3 A_{0}}{2+A_{0}} ; \quad \mu=\frac{2 A_{1}}{2+A_{0}} ; \quad v=\frac{2 A_{2}}{2+A_{0}} .
$$

Equation 2.6 shows that the Lam-Tung relation, $1-\lambda=2 v$, becomes $A_{0}=A_{2}$.

In the "naive" Drell-Yan the $q-\bar{q}$ axis coincides with the $\hat{z}$ axis of the Collins-Soper frame, hence $\theta_{1}=0$ and $\lambda=1$. The deviation of $\lambda$ from the "naive" Drell-Yan prediction of unity is due to non-zero $\theta_{1}$, which reflects the mis-alignment between the $q-\bar{q}$ axis and the $\hat{z}$ axis of the Collins-Soper frame [13, 14]. It is important to note that $\lambda$ (or $A_{0}$ ) does not depend on $\phi_{1}$, which is a measure of the non-coplanarity between the $q-\bar{q}$ axis and the hadron plane. In contrast, $\mu$ and $v$ (or $A_{1}$ and $A_{2}$ ) depend on both $\theta_{1}$ and $\phi_{1}$.

Equation 2.6 also shows that the Lam-Tung relation, $A_{0}=A_{2}$, is valid when $\phi_{1}=0$, i.e., for the co-planar case. Violation of the Lam-Tung relation is caused by the presence of the $\left\langle\cos 2 \phi_{1}\right\rangle$ term in $A_{2}$ (or $v$ ), and not due to the $A_{0}$ (or $\lambda$ ) term. Moreover, the non-coplanarity factor, $\left\langle\cos 2 \phi_{1}\right\rangle$, can be extracted from the data via the ratio $A_{2} / A_{0}$.

In perturbative QCD at the order of $\alpha_{s}$, ignoring the intrinsic transverse momenta of the colliding partons, the $q \bar{q} \rightarrow \gamma^{*} / Z G$ annihilation process gives [15, 16, 17]

$$
\left\langle\sin ^{2} \theta_{1}\right\rangle=\sin ^{2} \theta_{1}=q_{T}^{2} /\left(Q^{2}+q_{T}^{2}\right)
$$

in the Collins-Soper frame, where $q_{T}$ and $Q$ are the transverse momentum and mass, respectively, of the dilepton. One notes that $\theta_{1}$ given in Eq. 2.7 is identical to the angle between $\vec{P}_{B}$ (or $\vec{P}_{T}$ ) and the $\hat{z}$ axis in the Collins-Soper frame.

For the $q G \rightarrow \gamma^{*} / Z q$ Compton process, it was shown [5, 18, 19] that $\left\langle\sin ^{2} \theta_{1}\right\rangle$ is approximately described by

$$
\left\langle\sin ^{2} \theta_{1}\right\rangle=5 q_{T}^{2} /\left(Q^{2}+5 q_{T}^{2}\right),
$$

Using Eq. 2.6, the above two equations imply

$$
\begin{aligned}
\lambda & =\frac{2 Q^{2}-q_{T}^{2}}{2 Q^{2}+3 q_{T}^{2}} & v & =\frac{2 q_{T}^{2}}{2 Q^{2}+3 q_{T}^{2}} \\
\lambda & =\frac{2 Q^{2}-5 q_{T}^{2}}{2 Q^{2}+15 q_{T}^{2}} & v & =\frac{10 q_{T}^{2}}{2 Q^{2}+15 q_{T}^{2}}
\end{aligned}
$$

We note that for both processes, $\lambda=1$ and $\theta_{1}=0$ at $q_{T}=0$, while $\lambda \rightarrow-1 / 3$ and $\theta_{1} \rightarrow 90^{\circ}$ as $q_{T} \rightarrow \infty$. Moreover, Eq. 2.9 shows that the Lam-Tung relation, $1-\lambda=2 v$, is satisfied for both the $q \bar{q}$ and $q G$ processes at order $\alpha_{s}$. 


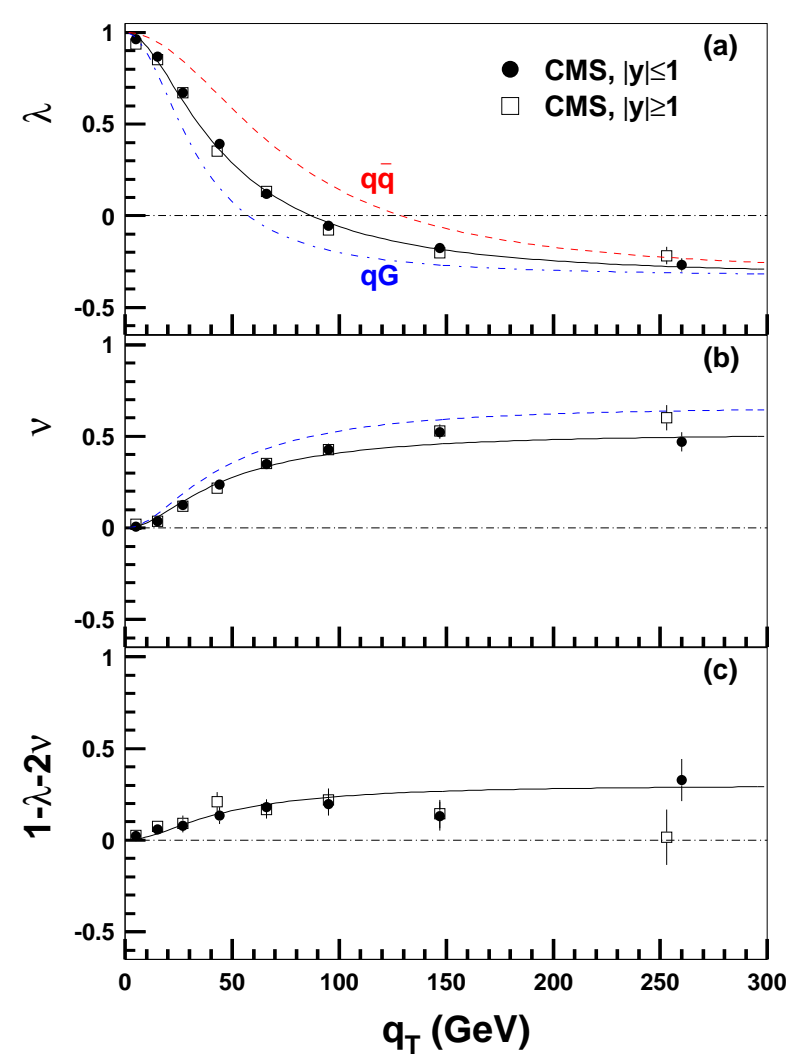

Figure 2: Comparison between the CMS data [10] on $\gamma^{*} / Z$ production at two rapidity regions with calculations for (a) $\lambda$ vs. $q_{T}$, (b) $v$ vs. $q_{T}$ (c) $1-\lambda-2 v$ vs. $q_{T}$. Curves correspond to calculations described in the text.

\section{Comparison with data}

The dashed and dash-dotted curves in Fig. 2(a) correspond to the calculation using Eq. 2.9 for the $q \bar{q}$ annihilation and the $q G$ Compton processes, respectively. Both the $q \bar{q}$ and $q G$ processes are expected to contribute to the $p p \rightarrow \gamma^{*} / Z X$ reaction, and the observed $q_{T}$ dependence of $\lambda$ must reflect the combined effect of these two contributions. A best-fit to the CMS data is obtained with a mixture of $58.5 \pm 1.6 \% q G$ and $41.5 \pm 1.6 \% q \bar{q}$ processes. The solid curve in Fig. 22(a) shows that the data at both rapidity regions can be well described by this mixture of the $q G$ and $q \bar{q}$ processes. In $p p$ collision the $q G$ process is expected to be more important than the $q \bar{q}$ process, in agreement with the best-fit result. While the amount of $q G$ and $q \bar{q}$ mixture can in principle depend on the rapidity, $y$, the CMS data indicate a very weak, if any, $y$ dependence. The good description of $\lambda$ shown in Fig. 2(a) also suggests that higher-order QCD processes are relatively unimportant.

We next consider the CMS data on the $v$ parameter. As shown in Eqs. 2.5 and 2.6, $v$ depends not only on $\theta_{1}$, but also on $\phi_{1}$. In leading order $\alpha_{s}$ where only a single undetected parton is present in the final state, the $q-\bar{q}$ axis must be in the hadron plane, implying $\phi_{1}=0$ and the Lam-Tung relation is satisfied. We first compare the CMS data, shown in Fig. Q $($ (b), with the calculation for $v$ using Eq. 2.9. which is obtained at the leading order $\alpha_{s}$. The dashed curve uses the same mixture 
of 58.5\% $q G$ and $41.5 \% q \bar{q}$ components as deduced from the $\lambda$ data. The data are at a variance with this calculation, suggesting the presence of higher-order QCD processes leading to a non-zero value of $\phi_{1}$. We performed a fit to the $v$ data allowing a non-zero value of $\phi_{1}$. The best-fit value is $A_{2} / A_{0}=0.77 \pm 0.02$. The solid curve in Fig. 2(b), corresponding to the best-fit, is in better agreement with the data. The non-zero value of $\phi_{1}$ also implies that the Lam-Tung relation is violated. This violation is indeed observed at CMS and shown explicitly in Fig. 2(c). The solid curve obtained with $A_{2} / A_{0}=0.77$ describes the observed violation of the Lam-Tung relation well.

The violation of the Lam-Tung relation reflects the non-coplanarity between the $q-\bar{q}$ axis and the hadron plane. This can be caused by higher-order QCD processes, where multiple partons are present in the final state in addition to the detected $\gamma^{*} / Z$. To illustrate this, one considers a specific quark-antiquark annihilation diagram at order $\alpha_{s}^{2}$ in which both the quark and antiquark emit a gluon before they annihilate. The hadron plane in this case is related to the vector sum of the two emitted gluons, and the $q-\bar{q}$ axis is in general not in the hadron plane. This would lead to a nonzero $\phi_{1}$ and a violation of the Lam-Tung relation. Similar consideration would also explain why the intrinsic transverse momenta of the colliding quark and antiquark in the "naive" Drell-Yan could also lead to the violation of the Lam-Tung relation, since the vector sum of the two uncorrelated transverse momenta would lead in general to a non-zero value of $\phi_{1}$.

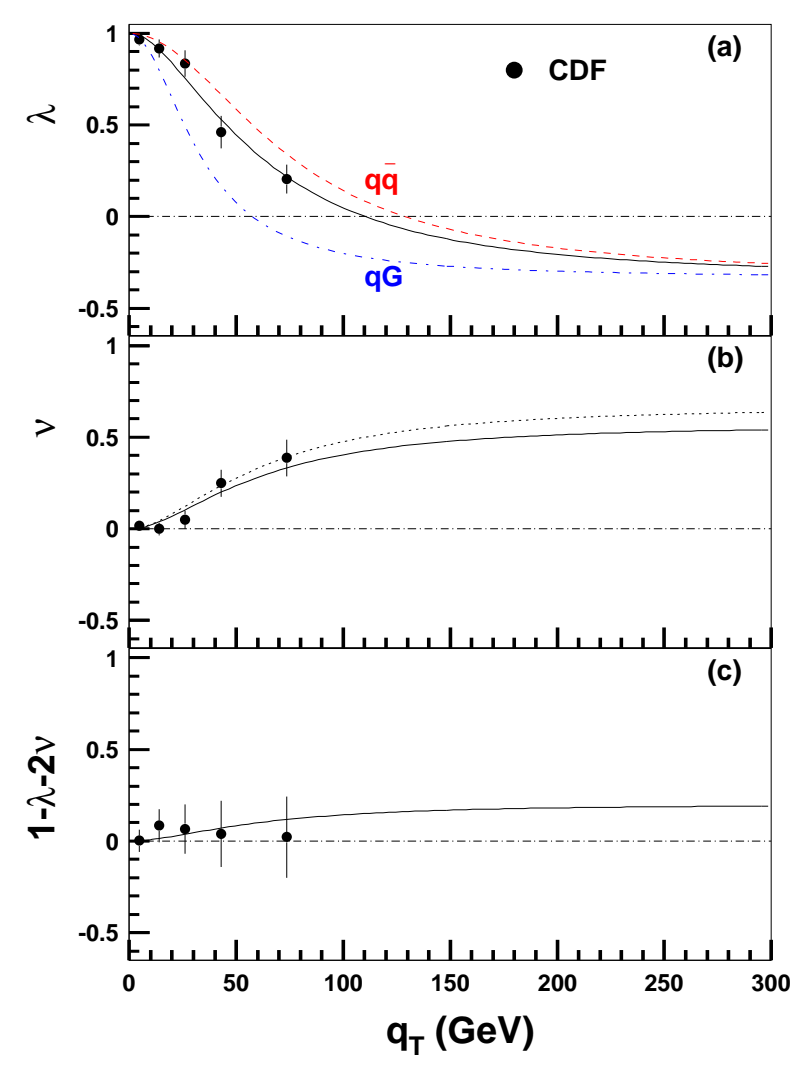

Figure 3: Comparison between the CDF data [9] on $\gamma^{*} / Z$ production with calculations for (a) $\lambda$ vs. $q_{T}$, (b) $v$ vs. $q_{T}$ (c) $1-\lambda-2 v$ vs. $q_{T}$. Curves correspond to calculations described in the text.

There remains the question why the $\mathrm{CDF} \bar{p} p$ Z-production data are consistent with the Lam- 
Tung relation [9]. Fig. 3 (a) shows $\lambda$ versus $q_{T}$ in $\bar{p} p$ collision at $1.96 \mathrm{TeV}$ from CDF. The $q_{T}$ range covered by the CDF measurment is not as broad as the CMS, and the statistical accuracy is somewhat limited. Nevertheless, a striking $q_{T}$ dependence of $\lambda$ is observed. The dashed and dash-dotted curves are calculations using Eq. 2.9 for the $q \bar{q}$ annihilation and the $q G$ Compton processes, respectively. The solid curve in Fig. 3 (a) shows that the CDF data can be well described with a mixture of $72.5 \% q \bar{q}$ and $27.5 \% q G$ processes. This is consistent with the expectation that the $q \bar{q}$ annihilation has the dominant contribution to the $\bar{p} p \rightarrow \gamma^{*} / Z X$ reaction. The CDF data on the $v$ parameter, shown in Fig. B.b), are first compared with the calculation (dotted curve) using Eq. 2.9 with a mixture of $72.5 \% q \bar{q}$ and $27.5 \% q G$ deduced from the $\lambda$ data. The solid curve in Fig. 3(b) results from a fit allowing $A_{2} / A_{0}$ to deviate from unity. The best-fit value is $A_{2} / A_{0}=0.85 \pm 0.17$. The relatively large undertainties The quantity $1-\lambda-2 v$, which is a measure of the violation of Lam-Tung relation, is shown in Fig. 3(c). The solid curve in Fig. B(c) is obtained using $A_{2} / A_{0}=0.85$. The CDF data is consistent with the solid curve, and the presence of some violation of the Lam-Tung relation can not be excluded by the CDF data.

\section{Conclusion}

We have presented an intuitive explanation for the observed $q_{T}$ dependencies of $\lambda$ and $v$ for the CMS and CDF $\gamma^{*} / Z$ data. The violation of the Lam-Tung relation can be attributed to the non-coplanarity of the $q-\bar{q}$ axis and the hadron plane, which occur for QCD processes involving at least two gluons. The present analysis could be further extended to the other coefficients, $A_{1}, A_{3}$ and $A_{4}$ [20]. It could also be extended [21] to the case of fixed-target Drell-Yan experiments, where the non-coplanarity at low $q_{T}$ can be caused by the intrinsic transverse momenta of the colliding partons in the initial states [20]. The effects of non-coplanarity on other inequality relations, as discussed in Ref. [22], are also being studied.

\section{References}

[1] S.D. Drell and T.M. Yan, Phys. Rev. Lett. 25, 316 (1970); Ann. Phys. (NY) 66, 578 (1971).

[2] I. R. Kenyon, Rep. Prog. Phys. 45, 1261 (1982).

[3] C.S. Lam and W.K. Tung, Phys. Rev. D18, 2447 (1978).

[4] C.S. Lam and W.K. Tung, Phys. Rev. D21, 2712 (1980).

[5] NA10 Collaboration, S. Falciano et al., Z. Phys. C31, 513 (1986); M. Guanziroli et al., Z. Phys. C37, 545 (1988).

[6] E615 Collaboration, J.S. Conway et al., Phys. Rev. D39, 92 (1989); J.G. Heinrich et al., Phys. Rev. D44, 1909 (1991).

[7] D. Boer, Phys. Rev. D60, 014012 (1999).

[8] Fermilab E866 Collaboration, L.Y. Zhu et al., Phys. Rev. Lett. 99, 082301 (2007); Phys. Rev. Lett. 102, 182001 (2009).

[9] CDF Collaboration, T. Aaltonen et al., Phys. Rev. Lett. 106, 241801 (2011).

[10] CMS Collaboration, V. Khachatryan et al., Phys. Lett. B750, 154 (2015). 
[11] J.C. Peng, W.C. Chang, R.E. McClellan, and O.V. Teryaev, Phys. Lett. B758, 384 (2016).

[12] J.C. Collins and D.E. Soper, Phys. Rev. D16, 2219 (1977).

[13] O.V. Teryaev, Proceedings of XI Advanced Research Workshop on High Energy Spin Physics, Dubna, 2005, pp. 171-175.

[14] P. Faccioli, C. Lourenco, J. Seixas, and H. Wohri, Phys. Rev. D83, 056008 (2011).

[15] J.C. Collins, Phys. Rev. Lett. 42, 291 (1979).

[16] D. Boer and W. Vogelsang, Phys. Rev. D 74, 014004 (2006).

[17] E.L. Berger, J.W. Qiu, and R.A. Rodriguez-Pedraza, Phys. Lett. B 656, 74 (2007).

[18] R.L. Thews, Phys. Rev. Lett. 43, 987 (1979).

[19] J. Lindfors, Phys. Scr. 20, 19 (1979).

[20] W.C. Chang, R.E. McClellan, J.C. Peng, and O.V. Teryaev, unpublished.

[21] M. Lambertsen and W. Vogelsang, Phys. Rev. D93, 114013 (2016).

[22] J.C. Peng, J. Roloff, and O.V. Teryaev, Proceedings of DSPIN 2012. 